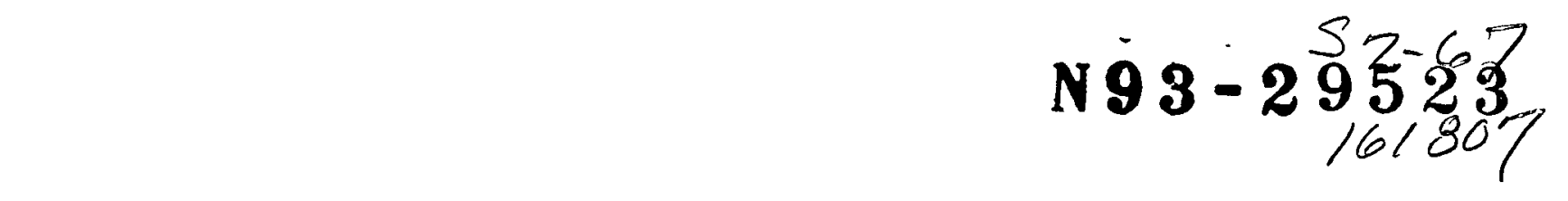

\title{
Quantitative Analysis of Properties and Spatial Relations of Fuzzy Image Regions
}

\author{
Raghu Krishnapuram, James M. Keller, and Yibing Ma \\ Department of Electrical and Computer Engineering \\ University of Missouri, Columbia, MO 65211 \\ phone: (314) 882 - 7766 \\ fax: (314) $882=0397$ \\ eceraghu@umcvmb.missouri.edu
}

\begin{abstract}
Properties of objects and spatial relations between objects play an important role in rulebased approaches for high-level vision. The partial presence or absence of such properties and relationships can supply both positive and negative evidence for region labeling hypotheses. Similarly, fuzzy labeling of a region can generate new hypotheses pertaining to the properties of the region, its relation to the neighboring regions, and finally, the labels of the neighboring regions. In this paper, we present a unified methodology to characterize properties and spatial relationships of object regions in a digital image. The proposed methods can be used to arrive at more meaningful decisions about the contents of the scene.
\end{abstract}

\section{Introduction}

The determination of properties of image regions and spatial relationships among regions is critical for higher level vision processes involved in tasks such as autonomous navigation, medical image analysis, or more generally, scene interpretation. In a rule-based system to interpret outdoor scenes, typical rules may be

\section{IF a REGION is THIN AND SOMEWHAT NARROW}

THEN it is a ROAD

IF a REGION is RATHER BLUE AND HOMOGENEOUS AND

IF THE REGION is $A B O V E$ a TREE REGION

THEN it is SKY

Although humans may have an intuitive understanding of words such as "thin" and "narrow", such concepts defy precise definitions, and they are best modeled by fuzzy sets. Similarly, humans are able to quickly ascertain the spatial relationship between two objects, for example " $B$ is above $A^{\prime \prime}$, but this has turned out to be a rather elusive task for automation. When the objects in a scene are represented by crisp sets, the all-or-nothing definition of the subsets actually adds to the problem of generating such relational descriptions. It is our belief that definitions of properties and spatial relationships based on fuzzy set theory, coupled with a fuzzy segmentation will yield realistic results.

Rosenfeld[1-3] defined many terms used in the analysis of spatial properties of objects represented by fuzzy sets. Pal has defined similar geometric attributes (such as index of area coverage) and have developed low- and intermediate-level algorithms based on such attributes [4]. Dubois and Jaulent[5] generalized Rosenfeld's definitions using both fuzzy set and evidence theories. 
Approximate spatial relation analysis has also attracted the attention of many researchers in the past several years. In many situations, precise description of relations among objects may be too complex and computationally too expensive. Approximate spatial relation analysis provides a natural way to solve real world problems with a reasonable cost. Freeman[6] was among the first to recognize that the nature of spatial relations among objects requires that they be described in an approximate(fuzzy) framework. Retz[7] has examined the intrinsic, deictic, and extrinsic use of spatial prepositions and has designed a system called CITYTOUR that answers natural language questions about spatial relations between objects in a scene and about the movement of objects. More recently, Dutta[8] has applied fuzzy inference and used a generalization of Warshall's algorithm to reason about object spatial positions and motion. However, modeling spatial relations among image objects is not addressed in any of these papers. Keller and Sztandera[9] addressed the problem of defining some spatial relationships between fuzzy subsets of an image by using dominance relations of projections of the regions onto coordinate axes.

In this paper, we propose direct methods to analyze properties of fuzzy image regions and spatial relations between fuzzy image regions quantitatively. The methods use membership functions generated by a fuzzy segmentation algorithm such as the fuzzy C-means algorithm [10]. The partition generated by the segmentation process is assumed to define $C$ fuzzy subsets, one representing each object or region in the image. We express the membership function of each object in terms of its $\alpha$-cut level sets and perform all computations on the level sets to obtain spatial properties of objects. We determine the relative positions of the level sets based on certain measurements on the elements of the level sets, and then we map the aggregated angle measurements into the interval $[0,1]$ using suitable membership functions to define spatial relations between regions as fuzzy sets over the domain of $\alpha$-levels.

In section 2, we describe methods to generate fuzzy subsets that describe the objects (regions) in the image. In section 3 , we review the existing methods to compute geometric properties and attributes of fuzzy image regions, and suggest how these methods can be easily extended to nongeometric properties and attributes. In section 4 , we describe our method to compute membership functions for spatial relations between fuzzy regions. The relations include LEFT OF, RIGHT OF, ABOVE, BELOW, BEHIND, IN FRONT OF, NEAR, FAR, INSIDE, OUTSIDE, and SURROUND. In section 5, we show some typical experimental results of attribute and spatial relation analysis involving fuzzy image regions. Section 6 contains the summary and conclusions.

\section{Generation of Fuzzy Subsets to Describe Objects in the Image}

Prewitt [11] was the first to suggest that the results of segmentation be fuzzy subsets of the image. In a fuzzy representation of an image, each object is represented by a fuzzy region $F$, where $F$ is defined over a referential set $\Omega$. Here, $\Omega$ is the domain over which the image function is defined. In this paper, we are mainly concerned with the discrete case, and hence $\Omega$ may be considered as a two-dimensional array. The membership function $\mu_{F}$ for the object is defined by: $\mu_{F}: \Omega \rightarrow[0,1]$. Each point $x=(x, y)$ in $\Omega$ is assigned a membership grade $\mu_{F}(x)$. It is further convenient to represent this region in terms of $\alpha$-cut level sets $F^{\alpha}$ as: $F^{\alpha}=\left\{x \mid \mu_{F}(x) \geq \alpha\right\}$, where $\alpha \in[0,1]$. In a real image, the number of membership values present is finite, and can be made quite small by quantizing the values. Hence, they can be enumerated as $1=\alpha_{1}>\alpha_{2}>\ldots>\alpha_{n}$. In

what follows, $\alpha_{n+1}$ will be assumed to be 0 . The level sets are nested, i.e., $F \alpha_{i \supseteq F} \alpha_{j}$ for $\alpha_{i}<\alpha_{j}$. 
In addition, for each $\alpha$-cut level set $F \alpha_{i}$, we can associate a basic probability assignment $m\left(F \alpha_{i}\right)$, where $m\left(F \alpha_{i}\right)$ satisfies: $\Sigma m\left(F^{\alpha_{i}}\right)=1$ [5].

One popular method for assigning multi-class membership values to pixels, for either segmentation or other types of processing, is the fuzzy C-means(FCM) algorithm [10]. The normalization of the memberships across classes in that approach sometimes leads to counterintuitive memberships. The partition generated by FCM may also be sensitive to noisy features and outliers. Also, the number of classes must be specified for the algorithm to run. The possibilistic $\mathrm{C}$ Means algorithm and the unsupervised clustering algorithms proposed by the authors overcome many of these problems [12-13].

\section{Properties and Attributes of Fuzzy Regions}

There are many ways to describe properties and attributes of an object. Properties and attributes of fuzzy image regions may be both geometric and non-geometric. In practical applications, some of the geometric properties that are frequently encountered are area, height, extrinsic diameter, intrinsic diameter, roundness, elongatedness, etc. [3]. Examples of nongeometric properties are brightness, color and texture. We now briefly summarize some geometric properties and their definitions from the existing literature [3].

The area of a fuzzy region $F$ is defined as the scalar cardinality of $F$, i.e.,

$$
a(F)=\sum_{x \in \Omega} \mu_{F}(x)
$$

The height $h$ of a fuzzy region $F$ along the direction $u$ is defined as

$$
h_{u}(F)=\sum_{u} \max _{v} \mu_{F}(u, v)
$$

where $v$ is the direction perpendicular to $u$. Rosenfield[2] defined the extrinsic diameter of a fuzzy region $F$ as

$$
E(F)=\max _{u} h_{u}(F)
$$

where $h_{u}$ is defined as above. The geometric property "elongatedness" may be defined in terms of the ratio of the minor extrinsic diameter and the major extrinsic diameter, i.e.,

$$
\mu_{\mathrm{EL}}(F)=1-\frac{\max _{u} h_{u}(F)}{E(F)}
$$

Conversely, the geometric property "roundness" may be defined as the complement of "elongatedness".

The geometric properties of objects can also be defined with respect to $\alpha$-cut level sets [5]. Assume we have nested $\alpha$-cut level sets $\left\{F^{\alpha_{1}} \subseteq F^{\alpha_{2}} \subseteq \ldots \subseteq F^{\alpha_{n}}\right\}$, with a basic probability assignment $m$ defined by

$$
m\left(F^{\alpha_{i}}\right)=\alpha_{i-} \alpha_{i+1},
$$

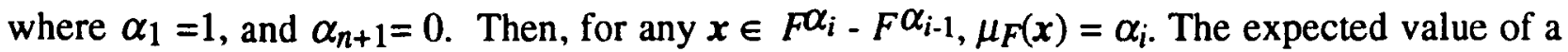
property $P(F)$, may be measured as:

$$
\tilde{P}(F)=\sum_{i=1}^{n} m\left(F \alpha_{i}\right) P\left(F \alpha^{\alpha}\right)=\sum_{i=1}^{n}\left(\alpha_{i-1} \alpha_{i+1}\right) P\left(F \alpha_{i}\right) .
$$


$\bar{P}(F)$ is the expected value of $P(F)$. Since $F \alpha_{i}$ is a crisp set, traditional techniques can be used to compute $P\left(F \alpha_{i}\right)$. For example, one may simple average the value of the property in the crisp region denoted by $F \alpha_{i}$ to obtain $P\left(F \alpha_{i}\right)$. Dubois and Jaulent proved [5] that $a(F)$ is the expected area $\bar{a}(F)$ and the height of $F$ along the $y$-axis is equal to the expected height along the $y$-axis of $F$. For the expected extrinsic diameter, the following inequality is true,

$$
\bar{e}(F) \geq E(F) \text {. }
$$

\section{Spatial Relations between Fuzzy Regions}

The primitive spatial relations between two objects are[6]: 1) LEFT OF, 2) RIGHT OF, 3) ABOVE, 4) BELOW, 5) BEHIND, 6) IN FRONT OF, 7) NEAR, 8) FAR, 9) INSIDE, 10) OUTSIDE, and 11) SURROUND. In he following, we present detailed definitions and methods for computation of memberships for some of the relations.

We define the relations as fuzzy sets over the universe of discourse of the $\alpha$-cut values $\left\{\alpha_{1}, \ldots, \alpha_{n}\right\}$. The general approach we use is as follows. Let $A$ and $B$ be two fuzzy sets defined on $\Omega$. At each $\alpha$-cut value $\alpha_{i}$, we compute the membership value for "A $\alpha_{i}$ RELATION $B^{\alpha_{i}}$ based on certain measurements $\gamma$ on the relative positions of the pairs of elements $(a, b), a \in A \alpha_{i}$ and $b \in B^{\alpha}{ }^{\alpha}$. These measurements are aggregated for all pairs elements to give an aggregated measurement $\gamma_{i}$. The membership value for " $A \alpha_{i}$ RELATION $B \alpha_{i}$ " denoted by $\mu_{A_{-} \text {REL_B }}\left(\alpha_{i}\right)$ is then computed by evaluating a membership function $\mu_{\mathrm{REL}}$ at $\gamma_{i}$. We are currently investigating methods to compute the overall membership for "A RELATION B", once the memberships for " $A \alpha_{i}$ RELATION $B \alpha_{i}$ " is computed for all $\alpha_{i}$. This may be achieved via a fuzzy aggregation operator, or from a method suggested by Dubois and Jaulent [5]. Ternary relations (such as "A IS BETWEEN $B$ and $C^{\prime \prime}$ ) can also be handled in a similar fashion.

In the following, we discuss the membership functions $\mu_{R E L}$ for some of the relations listed above in more detail. In Section 5, we show examples of membership computations for a variety of relations in different situations.

\subsection{LEFT OF}

Human perception of spatial positions between two objects is closely related to angular information. For example, one would search a sector area subtending an angle of approximately $180^{\circ}$ left of oneself to find an object that supposedly lies to one's left. Here, the distance between the person and the object is relatively unimportant. Based on this observation, we define most of the spatial relations in terms of angular measurements.

Suppose we have two points $A$ and $B$. Denote $A B$ as the line connecting $A$ and $B$. Let $\theta$ be the angle between $A B$ and the horizontal line, as shown in Figure 1. The membership function for " $A$ is to the LEFT of $B$ " may be defined as a function of $\theta$ as 


$$
\mu_{\mathrm{LEFT}}(\theta)=\left\{\begin{array}{cl}
1 & |\theta|<a \pi / 2 \\
\frac{\pi / 2-\mid \theta}{\pi / 2(1-a)} & a \pi / 2 \leq|\theta| \leq \pi / 2 \\
0 & |\theta|>\pi / 2
\end{array}\right.
$$
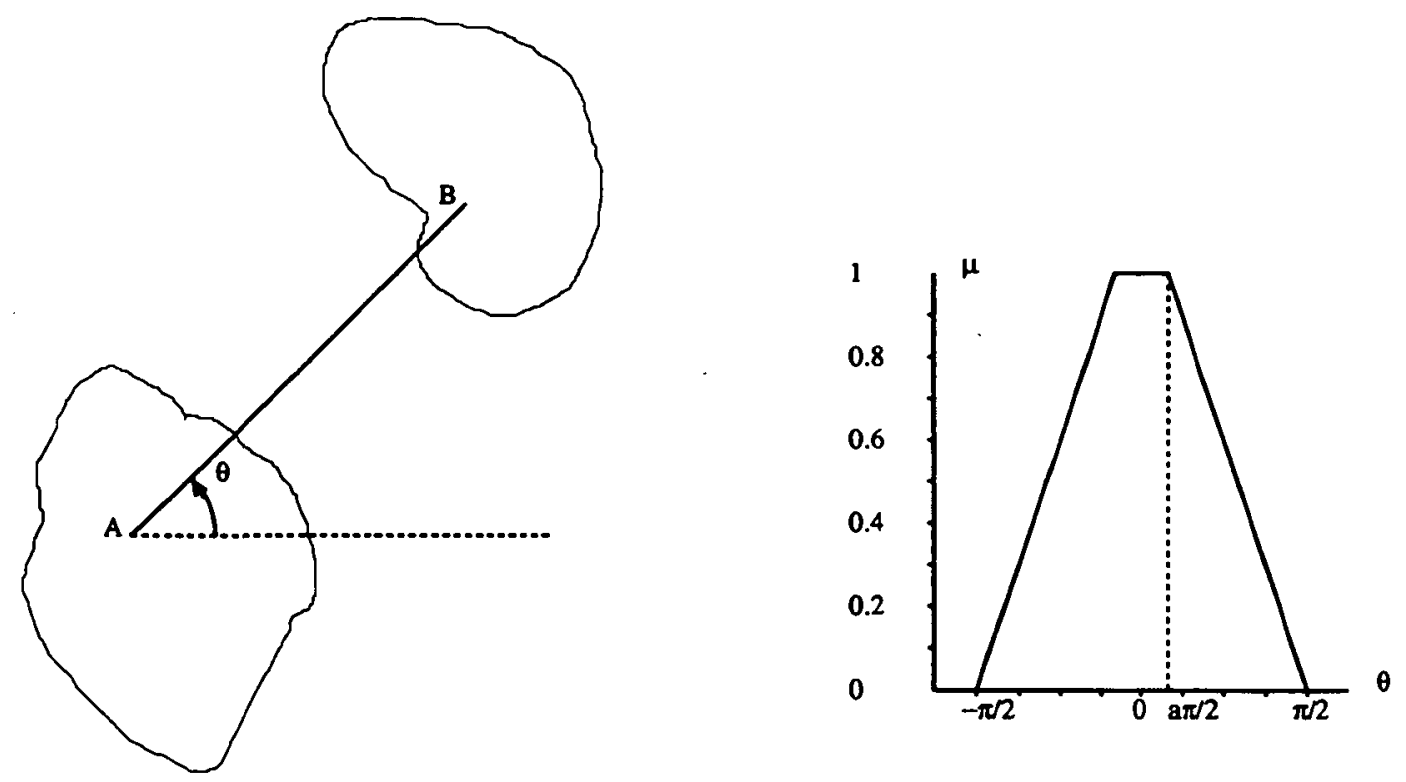

Figure 1: (a) Point relationship for "LEFT OF", (b) the membership function for "LEFT OF".

A large value for $a$ tends to give an optimistic result, and a small value would give a pessimistic result. Other symmetric functions may also be used to define $\mu_{\mathrm{LEFT}}$. The definition in (8) assumes that $A$ and $B$ are points. If they are two fuzzy regions, the angles described above are computed and averaged for all pairs of elements $(a, b), a \in \alpha_{i}$ and $b \in B \alpha_{i}$. The membership grade for "A $\alpha_{i}$ LEFT OF $B \alpha_{i}$ is obtained by mapping the averaged angle $\theta_{0}$ through the membership function defined in (8).

\subsection{RIGHT OF, ABOVE, BELOW, BEHIND, IN FRONT OF}

These relations may be calculated similar to the relation "LEFT OF", using aggregated values of angles made by lines joining pairs of points along with a corresponding trapezoidal membership function. Due to the symmetry in our definitions, the membership grade for " $A$ is to the LEFT OF $B$ " is the same as that for " $B$ is to the RIGHT of $A$ ". The symmetric property also applies to the relation pairs "ABOVE" - "BELOW", and "BEHIND" - "IN FRONT OF". It is to be noted that some of the terms mentioned above actually contain three dimensional information. As images are usually represented in a $2 \mathrm{D}$ space, some of these terms may not have any meaning.

\subsection{INSIDE, OUTSIDE}

For two level sets $A \alpha_{i}$ and $B \alpha_{i}$, the membership function for the spatial relation " $A \alpha_{i}$ is INSIDE $B \alpha_{i "}^{\prime \prime}$ may defined as, 


$$
\mu_{I N S I D E}\left(\alpha_{i}\right)=\frac{\mid A \alpha_{i} \cap B^{\alpha_{i} \mid}}{\mid B \alpha_{i \mid}},
$$

where $\mid A \alpha_{i \cap B} \alpha_{i \mid}$ and $\mid B \alpha_{i \mid}$ are the cardinalities of the level sets $A^{\alpha_{i}}$ and $B \alpha_{i}$ respectively. In a digital image, cardinality of a set is the number of pixels that belong to the corresponding level set. The membership function for "A is OUTSIDE B" can be defined as the complement of that for "A is INSIDE B".

\subsection{SURROUND}

If we assume that all the level sets of an object are connected regions, at each $\alpha$-cut level set, we can find two lines $l_{1}$ and $l_{2}$ for each point in $B^{\alpha_{i}}$, as shown in Figure 2 . Let $\theta$ denote the angle between the two lines as shown. The membership grade for " $A \alpha_{i}$ SURROUNDS $B \alpha_{i}$ " may be calculated by first computing the average $\theta_{i}$ of the angles $\theta$ for every element of $B \alpha_{i}$, and then applying the following mapping at $\theta=\theta_{i}$.

$$
\mu_{\text {SURROUND }}(\theta)=\left\{\begin{array}{cc}
1 & \theta>(2-a) \pi \\
\frac{\pi-\theta}{\pi(1-a)} & \pi \leq \theta \leq(2-a) \pi \\
0 & \theta<\pi
\end{array}\right.
$$

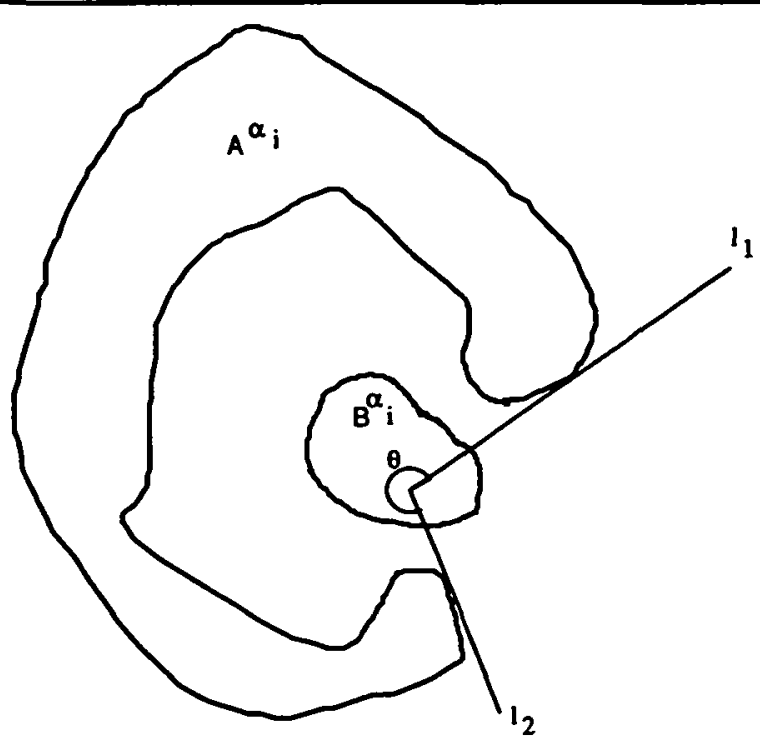

Figure 2: Definition of the angle $\theta$ to compute the relation "SURROUND"

\subsection{Spatial Relations among Objects (BETWEEN)}

Consider three points $A, B$ and $C$ as shown in Figure 3 . The membership value for " $C$ is BETWEEN $A$ and $B$ " may be defined using a trapezoidal shape as shown in Figure 3.

$$
\mu_{B E T W E E N}=\left\{\begin{array}{cl}
1 & |\theta-\pi| \leq a \pi / 2 \\
\frac{\pi /-|\pi-\theta|}{(1-a) \pi / 2} & a \pi / 2 \leq|\theta-\pi| \leq \pi / 2 \\
0 & |\theta-\pi| \geq \pi / 2
\end{array}\right.
$$


The membership value for " $C \alpha_{i}$ is BETWEEN $A \alpha_{i}$ and $B \alpha_{i}$ " may be computed by evaluating the membership function in (11) at $\theta=\theta_{i}$, where $\theta_{i}$ is the average of all the angles between lines $(a, c)$ and $(c, b)$, where $a \in A^{\alpha_{i}}$ and $b \in B^{\alpha_{i}}$ and $c \in C \alpha_{i}$. Other spatial relations among objects may be defined in a similar way.
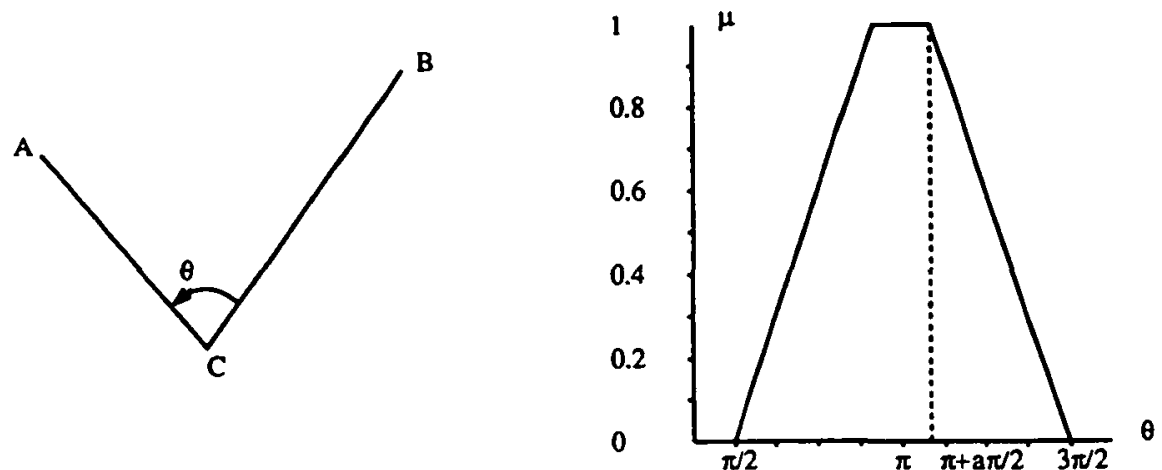

Figure 3: (a)Definition of the angle $\theta$ to compute the relation "BETWEEN", (b) the membership function for "BETWEEN".

\section{Examples of Spatial Relation Analysis}

Extensive simulations were conducted before we applied the proposed methods to real images. In the simulations, we chose objects with various membership function distributions, such as Gaussian shapes, triangular shapes, and exponential shapes. Relative positions and sizes of objects were also altered to observe the influence on the resulting membership functions for spatial relations. We first present two typical examples from our simulation experiments. We then present examples involving real images.
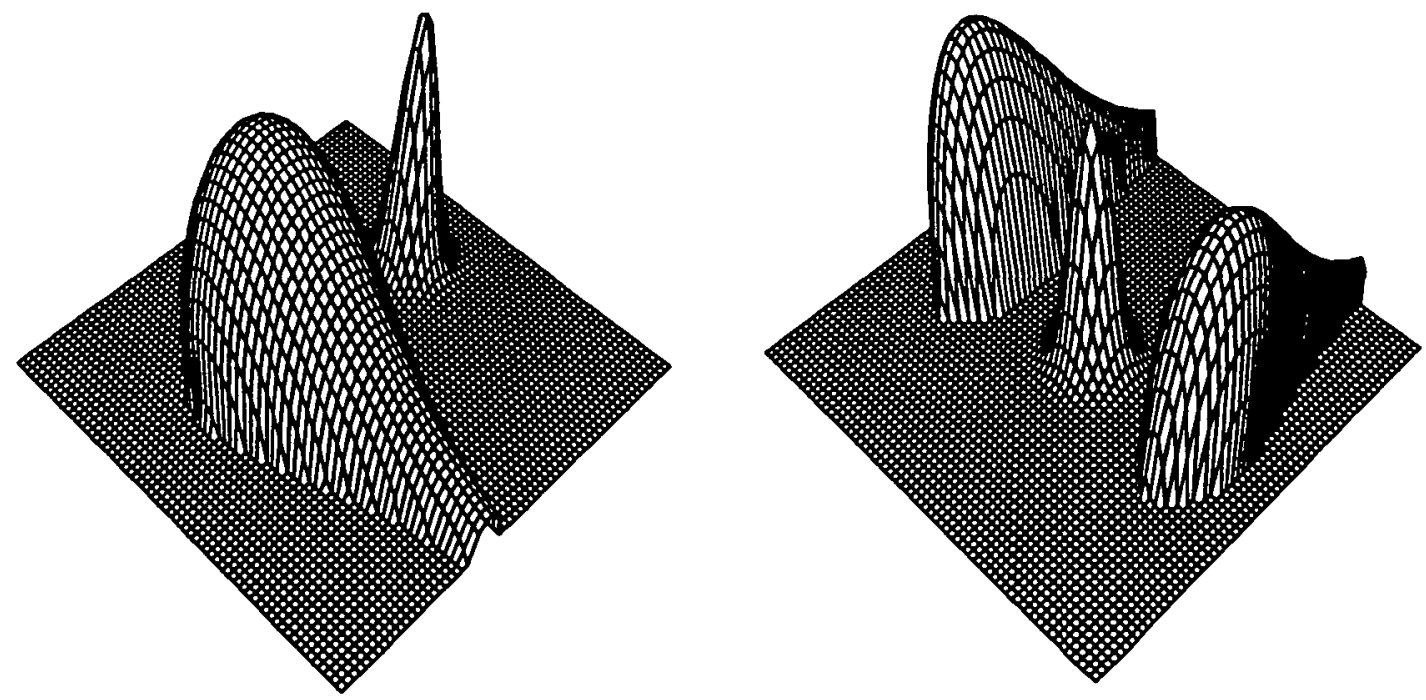

Figure 4: Synthetic membership functions for (a) two image regions, (b) three image regions. 
Figure 4(a) shows the fuzzy membership functions of two objects in an image and Figure 4(b) shows the fuzzy membership functions of three objects. The $z$-axis represents the membership grades for the objects. In Figure 4(a), the large object lies below the small object. In Figure 4(b), the small object lies in between the two large ones. It is to be noted that the membership functions for the large objects are not symmetric about the peak value. The membership grades of two spatial relations in the two images are shown in Figure 5. From Figure 5(a), we notice that at small $\alpha$-cut levels, object $A$ (large one) lies somewhat to the right of $B$. However, it is definitely below $B$. Therefore we have a reasonably high membership grade of about 0.85 for small $\alpha$-cut levels for the relation " $A$ is BELOW $B$ ". As the $\alpha$-cut level increases, object $A$ shrinks more and more to a position perfectly below object $B$. This results in a gradual increase of the membership grades to one. Similarly, in Figure 5(b), we initially have a low membership grade for $A$ is BETWEEN $B$ and $C$ and as the $\alpha$-cut level rises, object $A$ 's position is more BETWEEN $B$ and $C$. Therefore the membership grades related to the spatial relation also increases accordingly.

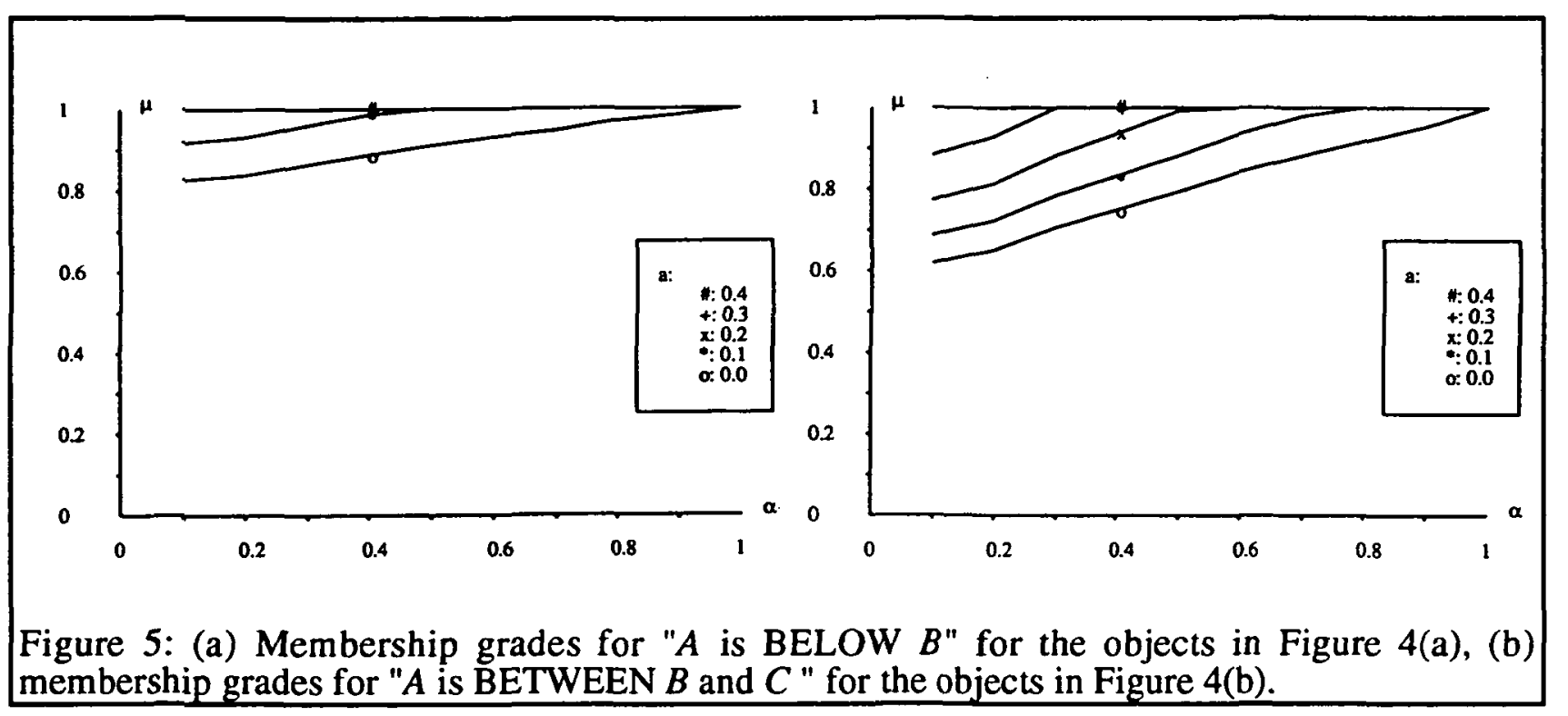

We next present some typical examples of our experimental results with real images. Figure 6 shows a $256 \times 256$ image of a natural scene as well as its segmentation by the GustafsonKessel algorithm [12]. (The closest crisp partition is shown.) A texture feature (homogeneity) and three color features (red, green, and blue) were used to perform the segmentation. The segmented image shows three main objects: sky, road, and trees. Figures 7(a) and 7(b) show the membership grades for the "correct" spatial relation "The sky is ABOVE the trees" and the "false" spatial relation "The sky is to the LEFT of the trees". In the image, a considerable portion of the sky is actually lower than the tree region. However, our method still generated high membership grades for the true hypothesis. This shows that our method of aggregating relations is very effective in capturing the intuitively correct overall spatial relation between regions. The membership grades for "The sky is to the LEFT of the trees" is low, as expected. Figure 7(e) shows the plot of the membership function for the ternary relation "The Trees are BETWEEN the SKY and the ROAD", for the segmentation shown in Figure 6(b). As expected, our method generated high membership grades for this correct hypothesis. 

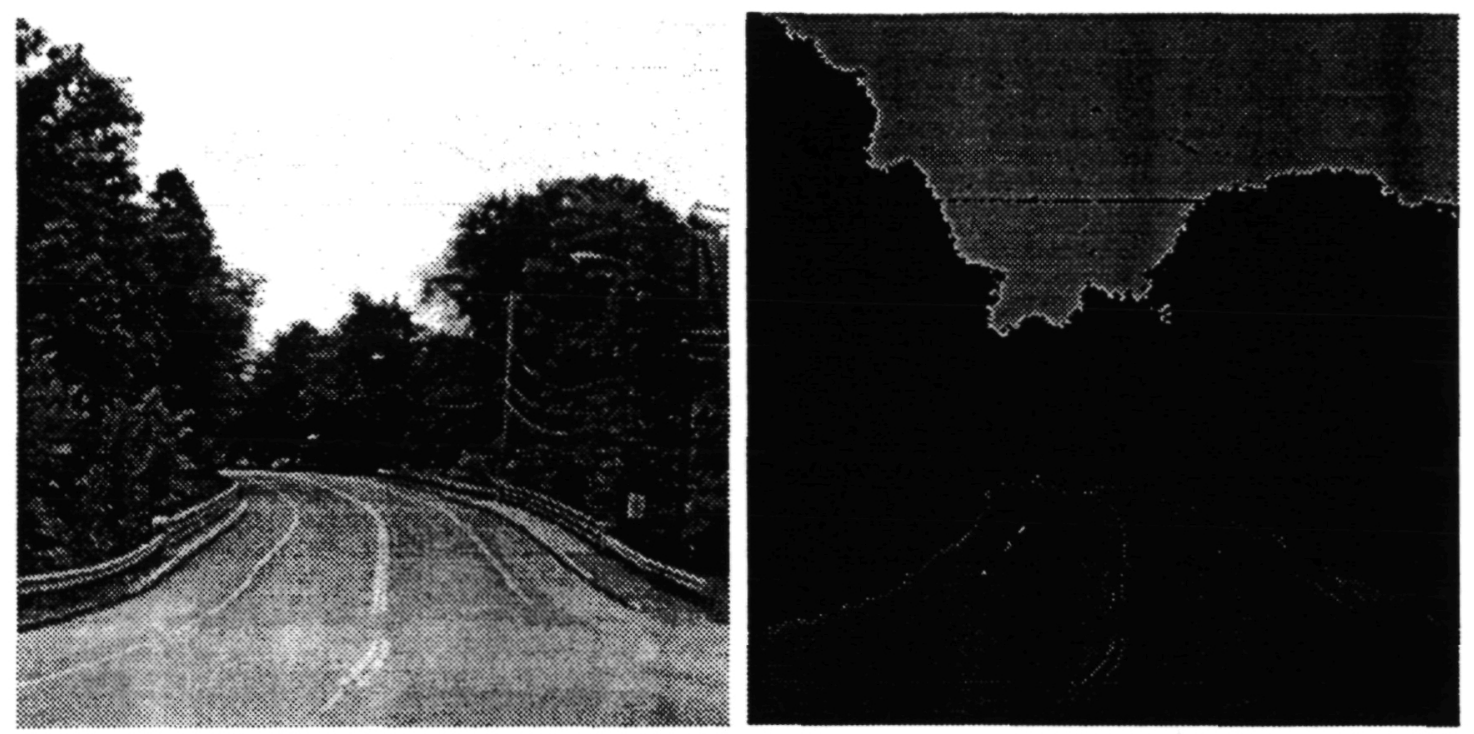

Figure 6: (a) Original $256 \times 256$ image of a natural scene, (b) the closest crisp partition of a segmentation.

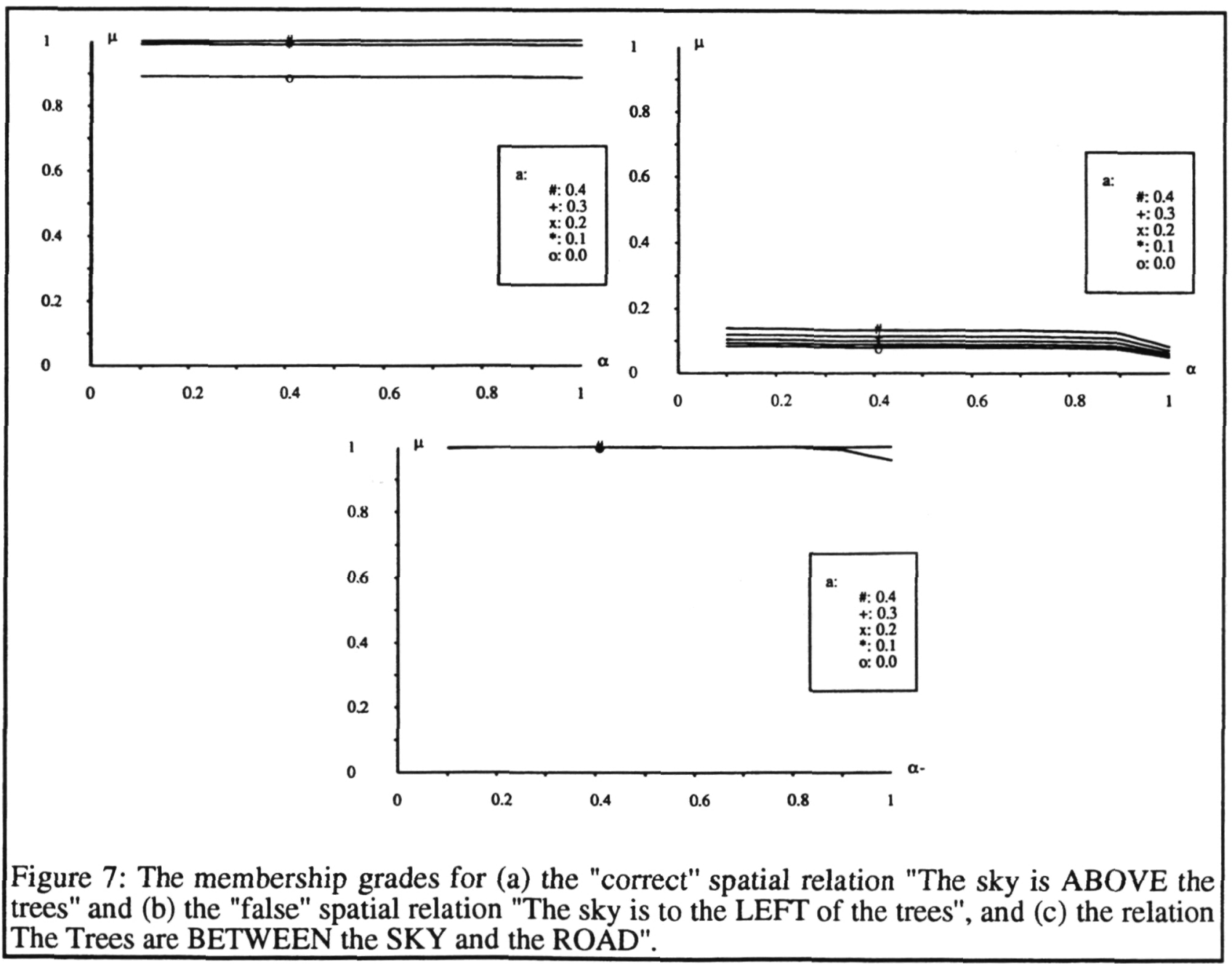




\section{Conclusions}

In this paper, introduce a new approach to analyze spatial relations between objects and among objects. In this approach, objects in the image are viewed as fuzzy regions, and spatial relations between fuzzy regions are viewed as membership functions (possibility distributions) defined over the set of $\alpha$-cut sets of the fuzzy regions. This $\alpha$-cut approach is similar to the approach introduced by Dubois and Jaulent ; and hence is consistent with the existing definition for the geometric properties of spatial regions. Since the properties and spatial relations are defined over the set of $\alpha$-cut sets, efficient algorithms to compute these relations can be devised, and these algorithms save considerable computation time. The methodology expressed in the paper can be widely used in such areas as image understanding, rule-based reasoning, and motion analysis.

\section{References}

1. A. Rosenfeld, "Fuzzy digital topology", Information and Control, Vol. 40, pp. 76-87, 1979.

2. A. Rosenfeld, "The fuzzy geometry of image subsets", Pattern Recognition Letters, Vol. 2, pp. 311-317, 1984.

3. A. Rosenfeld, "Fuzzy Geometry: An Overview", Proceedings of the First IEEE Conference on Fuzzy Systems, March 1992, San Diego, pp. 113-118.

4. S. K. Pal, "Fuzziness, image information and scene analysis", in An Introduction to fuzzy logic applications in intelligent systems, R. R. Yager and L. A. Zadeh (Ed.), Kluwer Academic, Norwell MA, 1992, pp. 147-184.

5. D. Dubois and M.-C. Jaulent, "A general approach to parameter evaluation in fuzzy digital pictures, Pattern Recognition Letters, Vol. 6, pp. 251-259, 1987.

6. J. Freeman, "The modeling of spatial relations", Computer Graphics and Image Processing, vol. 4, pp. 156-171, 1975.

7. G. Retz-Schmidt, "Various views on spatial prepositions", AI Magazine, Summer, 1988, pp. 95-105.

8. S. Dutta, "Approximate spatial reasoning: integrating qualitative and quantitative constraints", International Journal of Approximate Reasoning, vol. 5, pp. 307-331,1991.

9. J. Keller and L. Sztandera, "Spatial Relationships Among Fuzzy Subsets of an Image", Proc. Int. Symposium in Uncertainty Modeling and Analysis, Univ. of Maryland, December, 1990, pp. 207-211.

10. J. C. Bezdek, Pattern recognition with fuzzy objective function algorithms, Plenum Press, New York, 1981.

11. J. M. Prewitt, "Object enhancement and extraction", in Picture Processing and Psychopictorics, B.S. Lipkin and A. Rosenfeld (Eds.), Academic Press, New York, 1970, pp. 75-149.

12. R. Krishnapuram, and C.-P. Freg, "Fuzzy algorithms to find linear and planar clusters and their applications", Proceedings of the IEEE International Conference on Computer Vision and Pattern Recognition, Hawaii, June, 1991. pp.426-431.

13. R. Krishnapuram and J. M. Keller, "A possibilistic approach to clustering", submitted to the IEEE Transactions on Fuzzy Systems. 